\title{
A 100-Element Planar Schottky Diode Grid Mixer
}

\author{
Jonathan B. Hacker, Student Member, IEEE, Robert M. Weikle, II, Student Member, IEEE, Moonil Kim, Student
} Member, IEEE, Michael P. De Lisio, Student Member, IEEE, and David B. Rutledge, Senior Member, IEEE

\begin{abstract}
In this work we present a Schottky diode grid mixer suitable for mixing or detecting quasi-optical signals. The mixer is a planar bow-tie grid structure periodically loaded with diodes. A simple transmission line model is used to predict the reflection coefficient of the grid to a normally incident plane wave. The grid mixer power handling and dynamic range scales as the number of devices in the grid. A $10 \mathrm{GHz}$ 100-element grid mixer has shown an improvement in dynamic range of 16.3 to $19.8 \mathrm{~dB}$ over an equivalent single-diode mixer. The conversion loss and noise figure of the grid are equal to that of a conventional mixer. The quasi-optical coupling of the input signals makes the grid mixer suitable for millimeter-wave and submillimeter-wave applications by eliminating waveguide sidewall losses and machining difficulties. The planar property of the grid potentially allows thousands of devices to be integrated monolithically.
\end{abstract}

\section{INTRODUCTION}

$\mathrm{P}$ WER-COMBINING schemes involving solid-state devices quasi-optically coupled in free space have recently been employed to develop high-power microwave oscillators and amplifiers with directive beams [1]-[3]. These grid components lead the way to high-power solidstate millimeter-wave sources. However, oscillators and amplifiers are only two of many electronic components that are amenable to packaging in the grid configuration. A grid loaded with diodes produces a nonlinear device suitable for mixing or detecting quasi-optical signals with improved dynamic range compared to conventional single-diode mixers. This is particularly important for superconducting tunnel-junction (SIS) receivers where dynamic range is limited. Millimeter-wave high dynamic range front-ends are also the subject of a U.S. Navy initiative addressing current needs in its microwave electronics operational capability [4]. It should be possible to manufacture the grid mixer presented here as a planar monolithic circuit allowing a large number of diodes to be combined on a single wafer. This approach should give significant improvements in power-handling and dynamic range for mixers operating at millimeter-wave frequencies and above.

Manuscript received July 10, 1991; revised August 18, 1991. This work was supported by the Army Research Office and the Northrop Corporation. J. Hacker holds an NSERC Fellowship from Canada and M. De Lisio holds an NSF Fellowship.

The authors are with the Division of Engineering and Applied Science California Institute of Technology, Pasadena, CA 91125

IEEE Log Number 9105707.

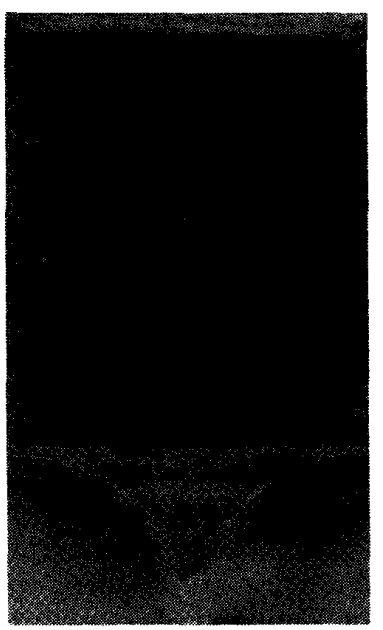

Fig. 1. The 100-Element planar Schottky diode grid mixer. The incident RF and LO electric fields are polarized vertically. The IF signal is taken off the top and bottom edges of the grid. The IF impedance of the square grid is equal to the IF impedance of a single diode. The diodes are bonded to the grid with conductive epoxy.

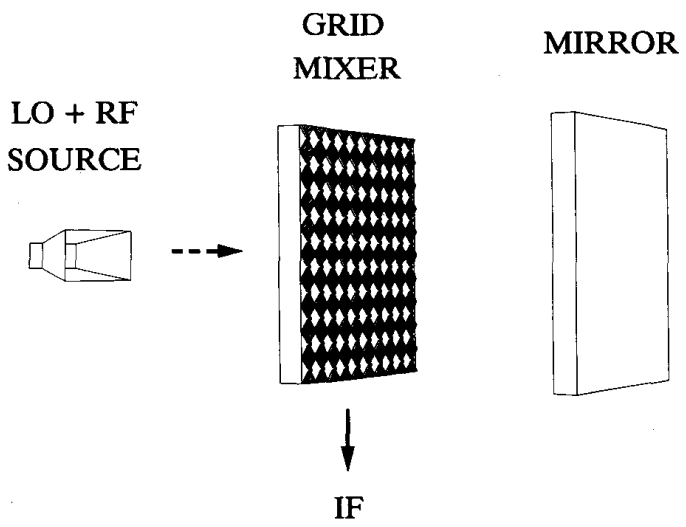

Fig. 2. Quasi-optical grid mixer configuration. The grid is mounted with the diodes facing the mirror. The incident RF and LO signals pass through the substrate which acts as an impedance transformer. The mirror is used to tune out the capacitive reactance of the Schottky diodes for a better match to free space.

The planar grid mixer is shown in Fig. 1. The devices are Hewlett-Packard low-barrier Schottky beam-lead diodes (HSCH-5332), suitable for mixers and detectors operating through the $K u$-band. The diodes are placed in a bow-tie shaped unit cell. The grid is fabricated on a 3.2 
$\mathrm{mm}$ thick Duroid substrate with $\epsilon_{r}=10.5$. The grid is 30 $\mathrm{mm}$ wide and the grid period is $3 \mathrm{~mm}$. There is a flat metal mirror behind the grid to act as a reactive tuning element (Fig. 2). The grid mixer reflection coefficient was optimized for incident signals near $10 \mathrm{GHz}$ by adjusting the dimensions of the unit cell bow-tie pattern and the substrate thickness. Diodes in each column are connected in series. The IF voltages add along each column and are collected at the diode terminals forming the top and bottom edges of the grid. A dc bias is also applied at the grid edges. The symmetry of the grid cancels any RF currents along the horizontal rows.

\section{Equivalent Circuit}

In an infinite grid with a uniform plane wave normally incident upon the grid surface, symmetry allows us to represent the grid as an equivalent waveguide unit cell. This waveguide has magnetic walls on the sides and electric walls on the top and bottom, as shown in Fig. 3. The walls extend in the $+z$ and $-z$ directions, with the diodes in the $z=0$ plane. In effect, this reduces the problem of analyzing the grid to that of analyzing an equivalent waveguide with electric and magnetic walls. The impedance presented to the terminals of a diode in the grid can be found by following a procedure similar to the EMF analysis in the paper by Eisenhart and Kahn [5]. This approach is justified by the fact that the grid period is only $\lambda / 10$. The calculations are described in detail by Weikle [6], and it is unnecessary to repeat them here. As a check on the EMF analysis, the grid was also modelled using the Hewlett-Packard High-Frequency Structure Simulator [7]. The HFSS is a three-dimensional full-wave electromagnetic solver that can be used to find the fields and $S$-parameters of an abritrary three-dimensional structure. The HFSS solution was in good agreement with the EMF analysis. The advantage of using the structure simulator over the EMF method is its ability to analyze arbitrary grid shapes that are too complicated for the EMF method.

For design purposes, we would like to find the reflection coefficient of an infinite grid for a plane wave at normal incidence on the front surface of the grid. The equivalent circuit for the grid mixer is shown in Fig. 4. The grid can be modeled as a three-port network. Ports 1 and 2 are respectively the front and back of the grid, and the third port is located between the two bow-ties, where the diode is mounted. The incident LO and RF TEM mode signals are modelled as a $377 \Omega$ source connected to port 1. The mirror placed behind the grid terminates port 2 in a short-circuited stub, and port 3 is terminated by the Schottky mixer diode. The entire grid is in this way reduced to a one-port equivalent circuit. A transmission line represents the propagating TEM mode as it passes through the substrate which supports the grid. The bow-tie grid is modeled as a short section of transmission line with characteristic impedance $Z_{B T}$ and electrical length $\theta_{B T}$. Values

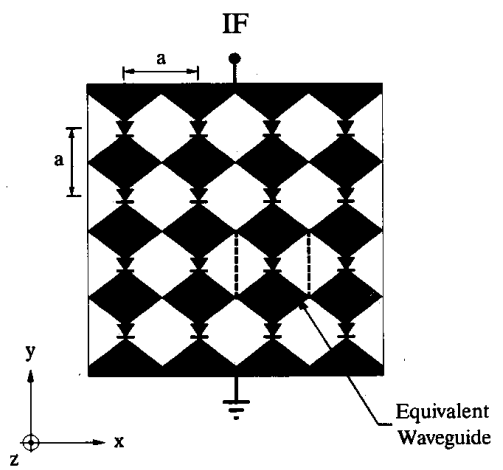

Fig. 3. Layout of the Schottky diode grid mixer. Boundary conditions are imposed by the grid symmetry. The solid lines are electric walls ( $E_{\text {tangential }}$ $=0$ ) and the dashed lines are magnetic walls $\left(H_{\text {tangential }}=0\right)$. In our grid, $a=3 \mathrm{~mm}$.

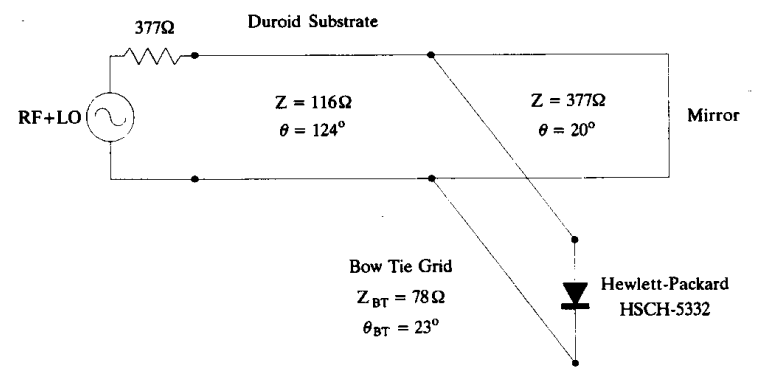

Fig. 4. Transmission line model for the grid mixer. The diode is modelled using the manufacturer's equivalent circuit. The reflection coefficient of the grid was matched to free space at the design frequency of $10 \mathrm{GHz}$.

for $Z_{B T}$ and $\theta_{B T}$ are obtained directly from the EMF analysis, or by parameter fitting to the HFSS simulation. The diode is added to the model by using the manufacturer's equivalent circuit. Simulation of the grid is carried out by calculating the reflection coefficient the grid presents to the RF and LO source connected to port 1 . The design was optimized by matching the grid to free space at the design frequency of $10 \mathrm{GHz}$.

The grid reflection coeffient was measured using a three-term error corrected quasi-optical reflectometer. The reflectometer consisted of a broadband horn placed inside an anechoic chamber and connected to an HP 8510 network analyzer. The grid was positioned approximately 0.5 $\mathrm{m}$ from the face of the horn during the measurement. The reflectometer was calibrated using three known standards, a short, a delayed short, and a matched load, in place of the grid. A mirror was used as the short, and a piece of absorber served as the matched load.

A measurement of the grid reflection coefficient using the quasi-optical reflectometer was found to be in reasonable agreement with theory as shown in Fig. 5(a). Fig. 5(b) shows the measured IF return loss. A dc bias current of $450 \mu \mathrm{A}$ per diode was required to match the IF impedance to $50 \Omega$. 


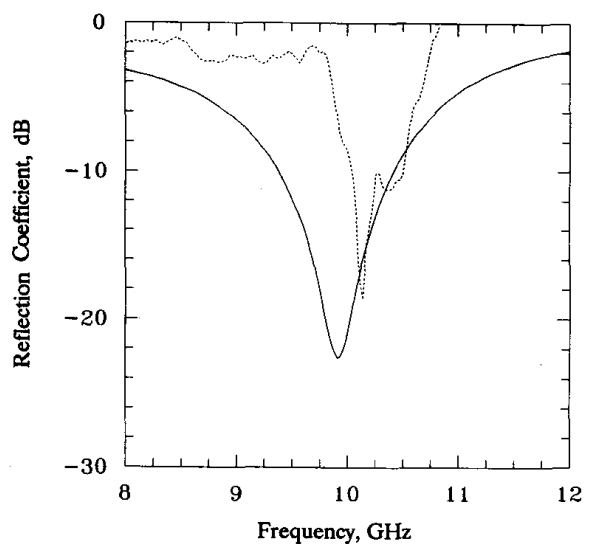

(a)

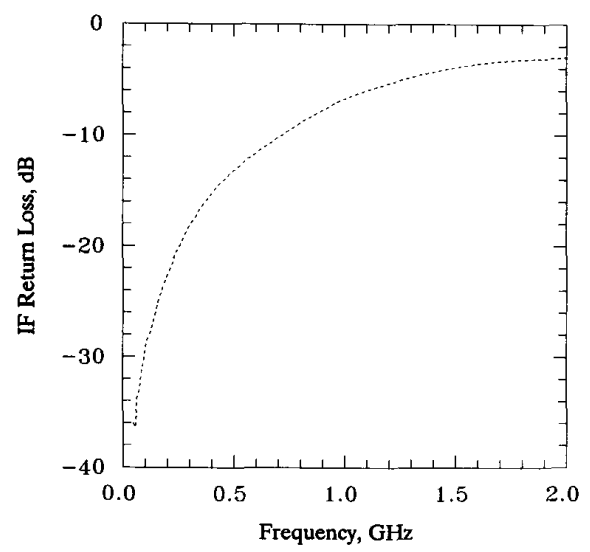

(b)

Fig. 5. (a) Theoretical (-) and measured (-- ) grid mixer reflection coefficient. The theoretical curve was obtained using the transmission line model developed for the grid, and the model provided by the manufacture of the Schottky diode. (b) Measured grid mixer IF return loss with a dc bias of $450 \mu \mathrm{A}$ per diode

\section{Conversion Loss AND Noise Figure}

For a square grid, the conversion loss of the grid mixer will be the same as a single-diode mixer in an equivalent embedding impedance. For the purpose of comparison, a single-diode microstrip mixer was designed and built with a diode of the same type used in the grid (Fig. 6). This allows us to compare the performance of the grid to that of an equivalent single-diode mixer. The microstrip mixer employs transmission line matching circuits for the RF/ LO and IF sections of the mixer [8]. The RF and LO signals were combined externally using a hybrid coupler to simplify the design. A dc bias was applied to the mixer diode through a bias-tee connected to the IF port.

The conversion loss of the grid mixer was defined as the power of the IF signal at the IF port divided by the total RF power incident upon the grid surface. For the conversion loss and noise figure measurements, the RF and LO signals were combined and fed to a single broadband horn. In order to accurately compute the incident RF and LO power densities at the grid surface, a calibration was made by removing the grid and placing an identical receive horn at twice the distance from the transmit horn. A measurement of the total system path-loss between the two horns with a network analyzer determined the horn gain and free space path-loss allowing an accurate computation of the power density at the grid surface. The power incident upon the grid was then defined as the product of the power density at the grid surface and the area of the grid. Thus, the grid conversion loss and noise figure measurements will also include any mismatch loss between the grid mixer and free space.

Fig. 7(a) shows the measured conversion loss of the grid mixer as a function of local oscillator power per diode for a combined $10.225 \mathrm{GHz}$ LO signal and a $10.439 \mathrm{GHz}$ RF signal normally incident upon the grid. Fig. 7(a) also shows the measured conversion loss of the equivalent single-diode microstrip mixer. The results verify that the grid mixer conversion loss is nearly equal to the single-diode mixer. The difference can be attributed to the slightly unequal impedances presented to the diodes for the two mixer designs. A grid conversion loss of $7.9 \mathrm{~dB}$ was measured for a local oscillator power of $-4 \mathrm{dBm}$ per diode.

The frequency response of the grid conversion loss is shown in Fig. 7(b) for a local oscillator power of -20 $\mathrm{dBm}$ per diode. Again, the performance of the equivalent single-diode mixer is included for comparison. The grid is band-limited by the reactive mirror tuning element. A more sophisticated design could exploit the broadband nature of the bow-tie grid by eliminating the mirror from the system. This would permit very broad bandwidths to be achieved.

The noise power of a square grid mixer is the same as the noise power of a single-diode mixer because the individual noise powers from each diode are uncorrelated. Consequently, the noise figure of the grid mixer will be the same as an equivalent single-diode mixer. In order to measure the noise figure of the grid, a Hewlett-Packard 8970 Noise Figure Meter was modified to allow quasioptical noise figure measurements (Fig. 8). The noise figure calibration was identical to that used for the grid mixer conversion loss measurement. The grid was placed in an anechoic chamber to shield the measurement system from external disturbances. Fig. 9 shows the measured noise figure of the grid for a local oscillator power of $-20 \mathrm{dBm}$ per diode. Again, the performance of the equivalent single-diode mixer is included for comparison. The results verify that the grid mixer noise figure and the single-diode mixer noise figure are nearly equal. The difference in noise figure can again be attributed to the slightly unequal impedances presented to the diodes for the two mixer designs. 


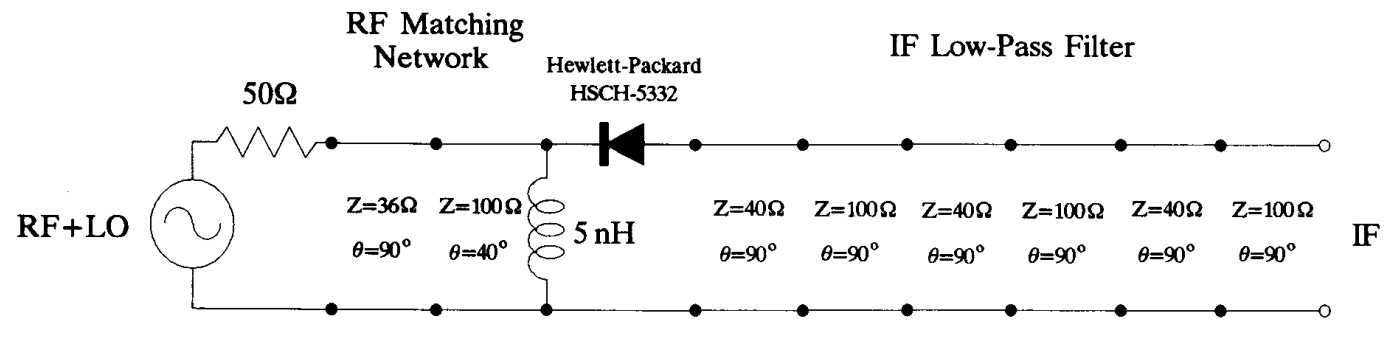

IF

Short

Fig. 6. Schematic of the microstrip mixer used for comparison with the grid mixer. The electrical lengths are specified at the LO design frequency. The IF low-pass filter provides a short at the RF/LO frequency. The lumped inductor shorts the RF/LO port at the IF frequency. The RF and LO signals are combined externally with a hybrid coupler. A dc bias was applied to the diode through a bias-tee connected to the IF port.

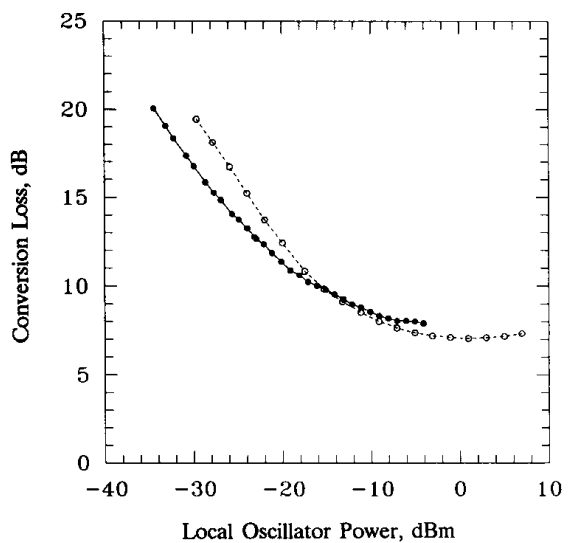

(a)

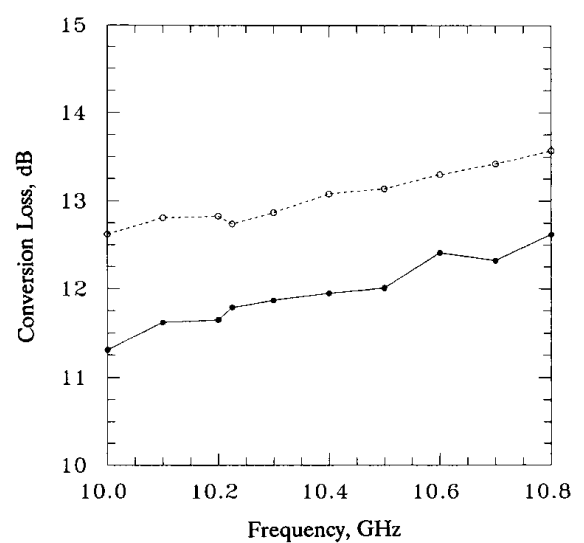

(b)

Fig. 7. (a) Measured grid mixer conversion loss (- - ) and equivalent single-diode mixer conversion loss (- - ) as a function of LO power per diode for an LO frequency of $10.225 \mathrm{GHz}$ and an IF frequency of $214 \mathrm{MHz}$. The conversion loss of the grid mixer is comparable to the single-diode mixer. (b) Measured grid mixer conversion loss ( $\longrightarrow$ - ${ }^{-}$and equivalent single-diode mixer conversion loss (--) as a function of frequency for a local oscillator power of $-20 \mathrm{dBm}$ per diode. The bandwidth of the grid mixer is primarily limited by the reactive tuning mirror.

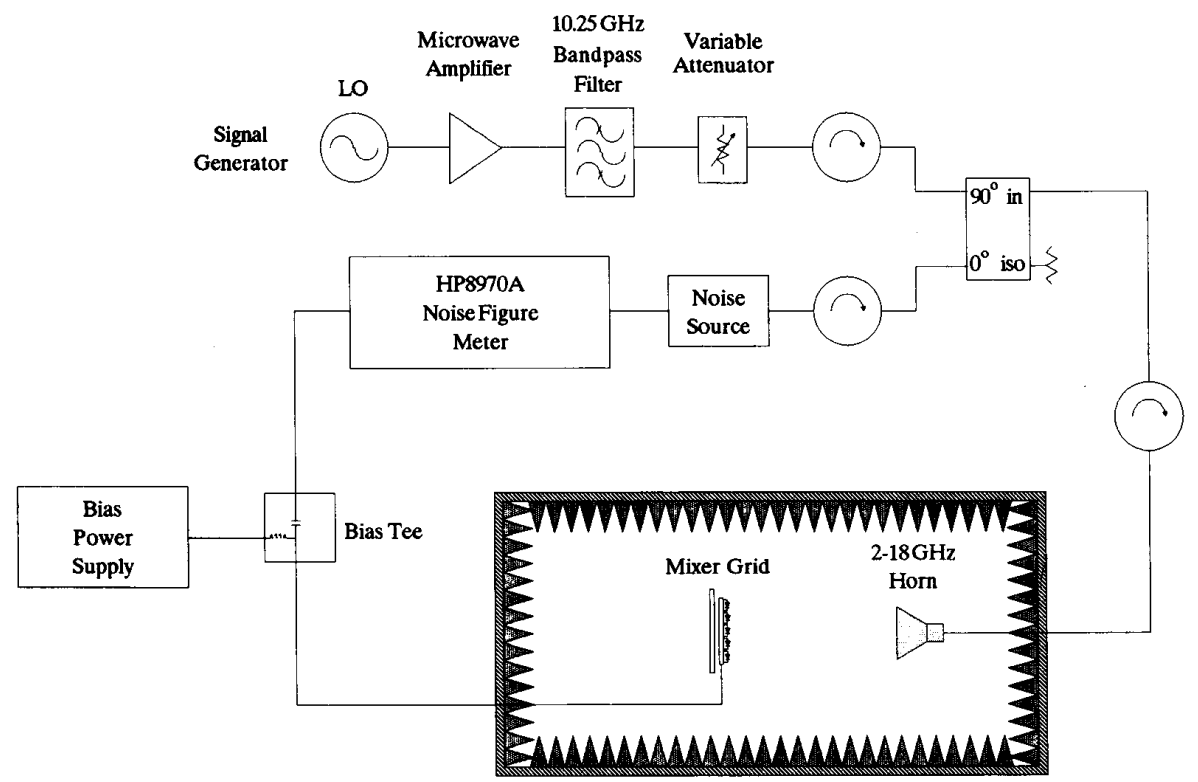

Fig. 8. Quasi-Optical Noise Figure Meter. A high temperature noise source is used to overcome the large system path-loss The microwave amplifier used was an HP $8349 \mathrm{~B}$ solid-state amplifier for LO powers below $-20 \mathrm{dBm}$ per diode, and a $10 \mathrm{~W}$ travelling wave tube amplifier for $L O$ powers above $-20 \mathrm{dBm}$ per diode. 


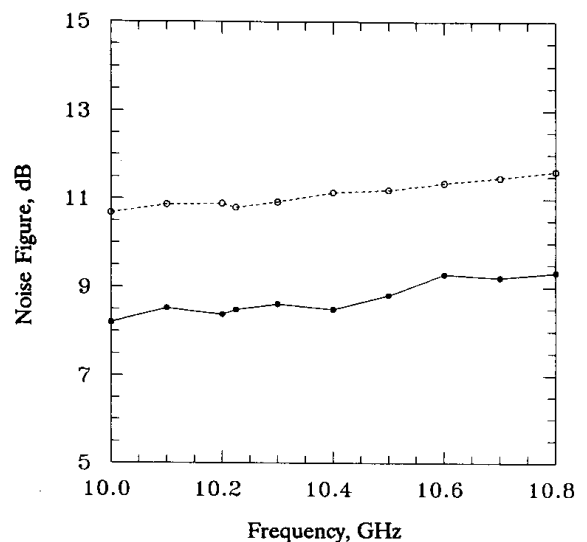

Fig. 9. Measured grid mixer noise figure $(-)$ and equivalent singlediode mixer noise figure (-- ) as a function of frequency for a local oscillator power of $-20 \mathrm{dBm}$ per diode. The noise figure of the grid mixer is comparable to that of a single-diode mixer. Excess noise from the TWT amplifier prevented swept frequency noise figure measurements for LO powers above $-20 \mathrm{dBm}$ per diode.

\section{Power Handling and Dynamic Range}

An important property of the grid mixer is its ability to increase dynamic range without compromising sensitivity. Since the RF power is spread among all the devices, the saturation power of the grid is increased by a factor of the number of devices. However, the noise figure of the grid remains equal to that of a single-diode mixer. Consequently, the dynamic range is increased by a factor of the number of devices in the grid as well. Of course, the tradeoff is that the required local oscillator power is also raised by the same amount. Unlike conventional mixers, though, the power handling of the grid can be increased without bound by increasing the number of diodes in the grid. At the same time, the conversion loss and noise figure of the grid can be independently optimized by adjusting the local oscillator power per diode. This decoupling of sensitivity and power handling makes the grid mixer particularly attractive for SIS mixer designs where power handling of the nonlinear element is fundamentally limited.

In order to measure the improvement in power handling of the grid, the linearity of the grid mixer was measured and compared to the single-diode mixer. The linearity of the mixer was characterized by computing the third-order intercept point of the mixer for two equal-power RF input tones separated by $10 \mathrm{MHz}$. For the same local oscillator power per diode, the grid mixer third-order intercept point should be 100 times larger than that of the single-diode mixer, a factor equal to the number of diodes in the grid. Fig. 10(a) shows the measured third-order intercept point for both the grid mixer and the single-diode mixer. Fig. 10(b) shows the difference in third-order intercept point for the two mixers. Improvements of 16.3 to $19.8 \mathrm{~dB}$ were measured over a $30 \mathrm{~dB}$ range of local oscillator powers. This compares favorably with the expected improvement of $20 \mathrm{~dB}$ predicted from theory for a 100 -element grid.

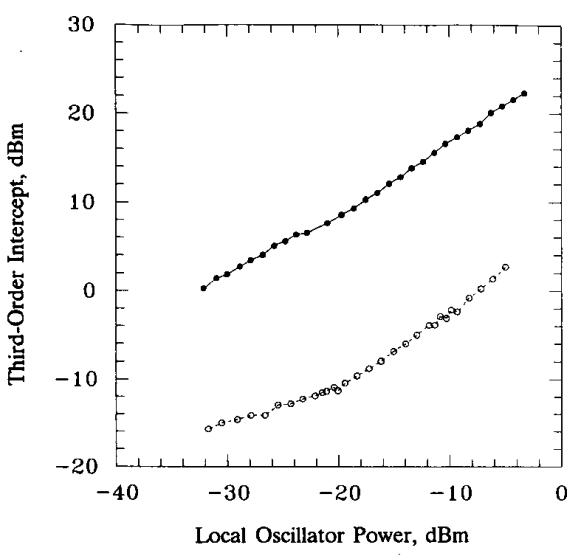

(a)

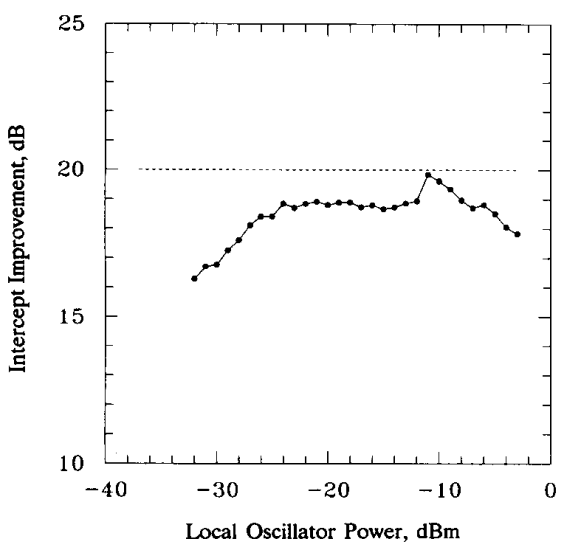

(b)

Fig. 10. (a) Measured grid mixer third-order intercept point $(-)$ and equivalent single-diode mixer third-order intercept point $(--)$ as a function of local oscillator power per diode. Two RF tones at $10.434 \mathrm{GHz}$ and $10.444 \mathrm{GHz}$ were used to measure the intermodulation products. (b) Measured improvement in third-order intercept point for the grid mixer over the single-diode mixer $(-)$ as a function of local oscillator power per diode. Theory (-- ) predicts a $20 \mathrm{~dB}$ improvement for a 100 -element grid.

\section{Conclusion}

In this paper we have presented a planar grid of 100 Schottky diodes suitable for use as a quasi-optically coupled mixer. We have developed a simple transmission line model for predicting the reflection coefficient of the grid to a normally incident plane wave. We have experimentally verified that the conversion loss and noise figure of the grid mixer are comparable to a conventional singlediode mixer. We have also verified that the power handling, and hence dynamic range, of the grid mixer increases in proportion to the number of diodes in the grid. It should be possible to make a monolithic grid mixer for operation at millimeter-wave frequencies. The mixer grid is attractive for millimeter-wave applications because of its low-loss quasi-optical coupling, and because its dynamic range can be increased by a factor of the number of devices in the grid. This is important for SIS receivers where dynamic range is fundamentally limited. 


\section{REFERENCES}

[1] Z. B. Popović, M. Kim, and D. B. Rutledge, "Grid oscillators," Int. J. Infrared and Millimeter-Waves, vol. 9, no. 7, pp. 647-654, July 1988.

[2] Z. B. Popović, R. M. Weikle, M. Kim, and D. B. Rutledge, "A 100MESFET planar grid oscillator," IEEE Trans. Microwave Theory Tech., vol. 39, no. 2, pp. 193-200, Feb. 1991.

[3] M. Kim et al., "A grid amplifier," IEEE Microwave Guided Wave Lett., vol. 1, no. 11, pp. 322-324, Nov. 1991.

[4] K. J. Sleger, R. H. Abrams, Jr., and R. K. Parker, "Trends in solidstate microwave and millimeter-wave technology," IEEE Microwave Theory Tech. Newsletter, no. 127, pp. 11-15, Fall 1990.

[5] R. L. Eisenhart and P. J. Kahn, "Theoretical and experimental analysis of a waveguide mounting structure," IEEE Trans. Microwave Theory Tech., vol. MTT-19, no. 8, pp. 706-719, Aug. 1971.

[6] R. M. Weikle II, "Quasi-optical planar grids for microwave and millimeter-wave power-combining," Ph.D. dissertation, California Institute of Technology, Pasadena, CA, 1992.

[7] "HP 85180A High-Frequency Structure Simulator," Hewlett-Packard Company, Networks Measurements Division, 1400 Fountaingrove Parkway, Santa Rosa, CA 95403.

[8] S. A. Maas, Microwave Mixers. Dedham, MA: Artech House, 1986, pp. 181-211.

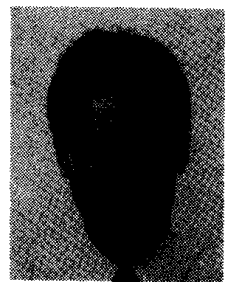

Jonathan B. Hacker (S'84) was born in Vancou ver, BC, Canada on February 16, 1963. He received the B.A.Sc. degree in electrical engineering in 1986 from the University of British Columbia, Vancouver, Canada, and the M.S. degree in electrical engineering from the California Institute of Technology, Pasadena in $1990 . \mathrm{He}$ is currently working toward the Ph.D. degree in electrical engineering at Caltech.

From 1986 to 1988 he was associated with the communications research and development group of the Nexus Engineering Corporation. His research interests include millimeter-wave quasi-optical techniques, microwave power amplifiers, and computer-aided design and measurement of microwave circuits.

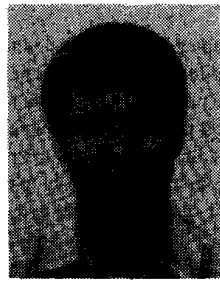

Robert M. Weikle, II (S'90) was born in Tacoma, WA, on February 13, 1963. He received the B.S. degree in electrical engineering and physics from Rice University, Houston, TX, in 1986 and the M.S. degree in electrical engineering from the California Institute of Technology in 1987. Currently he is working toward the Ph.D. degree at Caltech. His research interests include high-frequency solid-state devices and millimeter-wave quasi-optical techniques.

Mr. Weikle is a member of Phi Beta Kappa, Tau Beta Pi, and the American Physical Society.

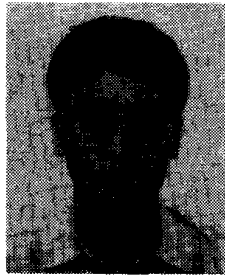

Moonil Kim (S'91) was born in Seoul, Korea, on March 14, 1965. He received the B.S degree from the Illinois Institute of Technology in 1987, and the M.S. degree from the California Institute of Technology in 1988 . He is currently working toward the Ph.D. degree in the MMIC group at Caltech. His research interests include quasi-optical millimeter power combining components such as grid oscillators and amplifiers.

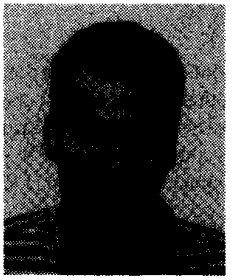

Eta Kappa Nu.
Michael P. De Lisio (S'90) was born in Detroit, MI, on July 29,1968 . He received the B.S.E. degree in electrical engineering from the University of Michigan, Ann Arbor, in 1990. In 1991, he obtained the M.S. degree from the California Institute of Technology. He is currently pursuing the Ph.D. degree at the California Institute of Technology. Monolithic methods of millimeter-wave power combining and high-frequency solid-state devices are some of his research interests.

Mr. De Lisio is a member of Tau Beta Pi and

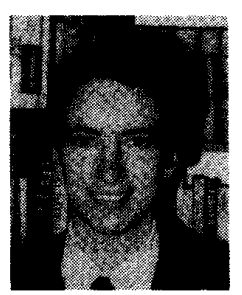

David B. Rutledge (S'75-M'80-SM'89) was born in Sayannah, GA on January 12,1952 . He re ceived the B.A. degree in mathematics from Williams College, Williamstown, MA, in 1973, the M.A. degree in electrical sciences from Cambridge University, Cambridge, England, in 1975, and the $\mathrm{Ph} . \mathrm{D}$. degree in electrical engineering from the University of California at Berkeley in 1980 .

In 1980 he joined the faculty at the California Institute of Technology, Pasadena, $\mathrm{CA}$, where he is now Professor of Electrical Engineering. His research is in developing millimeter and submillimeter-wave monolithic integrated circuits and applications, and in software for computer-aided design and measurement. Dr. Rutledge is co-author of the software CAD program, Puff, which has over 8000 users worldwide. 